\title{
Effects of Gentiopicroside on activation of NLRP3 inflammasome in acute gouty arthritis mice induced by MSU
}

\author{
Menglin $\mathrm{He}^{1} \cdot$ Cheng $\mathrm{Hu}^{1} \cdot$ Meijuan $\mathrm{Chen}^{2} \cdot \mathrm{Qian}_{\mathrm{Gao}^{2}} \cdot \mathrm{Liqiu}_{\mathrm{Li}}^{2} \cdot$ Weiqian Tian ${ }^{1} \mathbb{C}$
}

Received: 25 May 2021 / Accepted: 20 September 2021 / Published online: 29 September 2021

(c) The Author(s) 2021

\begin{abstract}
Acute gouty arthritis is a self-limiting inflammatory disease resulting from the deposition of monosodium urate (MSU) crystals. It has been shown that Gentiopicroside (GPS) possesses anti-inflammatory and analgesic functions. The aim of this study was to parse out whether GPS has an effect on acute gouty arthritis. We established an acute gouty arthritis model by the injection of MSU into the paw, and found that GPS relieves MSU-induced mechanical, thermal hyperalgesia, and paw swelling. Furthermore, GPS down-regulated the release of pro-inflammatory cytokines in paw tissues, including IL-1 $\beta$, IL-6, IL-18, and TNF- $\alpha$. The results of H\&E staining and MPO activity measurement showed that GPS inhibits neutrophil infiltration. And the over-expressions of NOD-like receptor protein 3 (NLRP3), apoptosis-associated speck-like protein containing a caspase recruitment domain (ASC), and Caspase-1 induced by MSU were inhibited by treatment with GPS. These results revealed that GPS can treat acute gouty arthritis based on anti-inflammatory and analgesic properties in vivo, which might be ascribed to the inhibition on NLRP3 inflammasome. Furthermore, we performed in vitro study to confirm the results of in vivo study. Consistently, the results proved that GPS could inhibit the activation of NLRP3 inflammasome in RAW264.7 macrophages stimulated by LPS-MSU. In conclusion, this study provides an experimental basis for the application of GPS and expands the potential value of GPS in the therapy of acute gouty arthritis.
\end{abstract}

Keywords Gentiopicroside $\cdot$ Inflammation $\cdot$ Acute gouty arthritis $\cdot$ NLRP3 inflammasome

\section{Introduction}

Gout is a common and reversible auto-inflammatory disease, which is characterized by the disorder of the innate immune and causes systemic inflammatory response [1]. It has been reported that gout is the most conventional type of inflammatory arthritis globally [2]. Uric acid is a physiological substance in our body, which has an antioxidant ability in low concentration [3]. However, high concentration of urate is identified as the key factor required for MSU

Menglin He and Cheng Hu contributed equally to this work.

Weiqian Tian

twq1972@163.com

1 Department of Anesthesiology, Affiliated Hospital of Nanjing University of Chinese Medicine, Jiangsu Province Hospital of Chinese Medicine, No. 155 Hanzhong Road, Qinhuai District, Nanjing 210029, Jiangsu, China

2 School of Medicine and Holistic Integrative Medicine, Nanjing University of Chinese Medicine, Nanjing 210023, Jiangsu, China crystallization [4]. And some patients with the deposition of MSU crystals manifest gout flare with symptoms of pain, swollen, and hot joint [5]. Additionally, the joints affected by gout are characterized by obvious neutrophil infiltration [6]. Multiple medications have been applied to treat acute gouty arthritis, including NASIDs, glucocorticoid and colchicine. But these treatments have inevitable limitations in clinic [7].

MSU crystals are first phagocytized by macrophages, and then promote the assembly and activation of NLRP3 inflammasome [8]. The activation of NLRP3 inflammasome needs NLRP3 to recruit the adaptor ASC, which then recruits caspase-1, resulting in the formation of inflammasome complex ultimately [9]. As an effector, caspase- 1 cleaves the pro-IL- $1 \beta$ and pro-IL- 18 into IL- $1 \beta$ and IL-18, respectively [10]. Furthermore, IL-1 $\beta$ elevates the expression of genes, which are related to fever, pain threshold, and promote immune cells to infiltrate infected or damaged tissues [11]. And IL-18 is a co-stimulatory cytokine that regulates adaptive immunity [12]. Research [13] demonstrated that multiple pro-inflammatory cytokines, such as IL- 6 and TNF- $\alpha$, are related to gouty arthritis. Therefore, the inhibition on 
NLRP3 inflammasome and the release of pro-inflammatory cytokines are crucial targets in the treatment of acute gouty arthritis.

GPS, as an iridoid glucoside separated from the root of Gentiana Macrophylla Pall, has been widely used to cure several diseases based on anti-inflammatory, hepatoprotective, and anti-cancer properties [14-16]. GPS has been reported to inhibit the inflammation response in rat articular chondrocytes induced by IL-1 $\beta$ [17] and colitis in mice induced by dextran sodium sulfate [18]. Research [19] also demonstrated that GPS could inhibit the secretion of inflammatory factors. Furthermore, a recent study has studied the protective effect of GPS on ethanol-induced gastric mucosal injury, and the mechanism of effect was related to anti-inflammatory [20].

The effects of GPS have been illustrated in the above findings explicitly. However, the relation between GPS and acute gouty arthritis remains unknown. Therefore, the present study aimed to shed the light on the mechanism of GPS in treating acute gouty arthritis. We evaluated the effects of GPS on pain, paw swelling, and neutrophil infiltration in MSU-induced acute gouty arthritis mice. The inhibition of GPS on the release of pro-inflammatory cytokines was also explored. Furthermore, the activation of NLRP3 inflammasome was analyzed both in vivo and vitro studies.

\section{Materials and methods}

\section{Experimental animals}

Specific-pathogen-free male C57BL/6 mice (18-22 g, 6-8 weeks of age) were purchased from Qinglong Mountain Animal Breeding Farm, Nanjing, China. The mice were housed in plastic cages and kept on a 12/12 h light/dark cycle and were given access to food and water with no restrictions in a room with an ambient temperature of $22{ }^{\circ} \mathrm{C} \pm 2{ }^{\circ} \mathrm{C}$ during the experiment. All animal procedures were carried out in strict accordance with the guideline of the National of Health Guide for the Care and Use of Laboratory Animals.

\section{Cell culture and treatment}

RAW264.7 macrophage cell line was purchased from Shanghai Fuheng Biology (Shanghai, China) and cultured in DMEM (Gibco, USA) supplemented with 10\% FBS (Hycolon, USA) at $37{ }^{\circ} \mathrm{C}$ with $5 \% \mathrm{CO}_{2}$ and $95 \%$ air. After preconditioning with or without LPS at the concentration of 200 $\mu \mathrm{g} / \mathrm{ml}$ for $4 \mathrm{~h}$, the cells were treated with GPS $(1250,2500$, and $5000 \mu \mathrm{M}$ ) followed by exposure to $500 \mathrm{ug} / \mathrm{ml}$ MSU for $4 \mathrm{~h}$. LPS, GPS, and MSU were dissolved in PBS, and MSU solution was processed with Ultrasonic Liquid Processors before application (QSONIC, USA).

\section{Cell viability assay}

The Cell Counting Kit- 8 was applied to assess the cytotoxicity of GPS. Primarily, $5 \times 10^{3}$ RAW264.7 cells were plated per well of a 96-well plate followed by $24 \mathrm{~h}$ incubation. Then cells were treated with a range of GPS concentrations for another $24 \mathrm{~h}$ in $100 \mu \mathrm{L}$ medium. The medium was removed, CCK-8 reagent and fresh medium were added to per well at $1: 10$ ratio in a $2 \mathrm{~h}$ incubation period at $37^{\circ} \mathrm{C}$. The absorbance in $450 \mathrm{~nm}$ was measured spectrophotometrically using a microplate reader (BioTek, Santa Barbara, CA, USA).

\section{Reagents}

GPS (purity $>97 \%$, \#20831-76-9) was purchased from Yuanye Bio-Technology (Shanghai, China). GPS was dissolved in PBS as a stock solution at $1 \mathrm{~g} / \mathrm{ml}$ and stored at $4{ }^{\circ} \mathrm{C}$ before use. Lipopolysaccharide (LPS) (L2630) was purchased from Sigma-Aldrich (USA). Antibodies against NLRP3 (AG-20B-0014-C100) and Caspase-1 (AG-20B-0042-C100) were purchased from Adipogen Life Sciences (San Diego, USA). Antibodies against ASC (\#67824) and $\beta$-actin (\#3700) were obtained from Cell Signaling Technology (Cell Signaling, Danvers, Technology, MA, USA). Horseradish peroxidase (HRP)-conjugated goat anti-mouse (SA00001-1), and HRP-conjugated goat anti-rabbit (SA00001-2) antibodies were purchased from proteintech (Boston, MA, USA). The BCA Protein Assay Kit was obtained from Beyotime (Beyotime Institute of Biotechnology, Nanjing, China).

\section{Preparation of MSU crystals}

Uric acid (800 mg) was added into boiling water $(155 \mathrm{~mL})$ containing $1 \mathrm{~N} \mathrm{NaOH}(5 \mathrm{~mL})$. And the $\mathrm{PH}$ of solution was altered to 7.2 with hydrochioric acid $(\mathrm{HCl})$. MSU crystals were prepared after autoclaved sterilization. The whole procedures followed the previous study [21]. The structure of MSU crystals was visualized (Fig. 1a).

\section{Establishment and treatment of acute gouty arthritis model}

48 male C57BL/6 mice were randomly divided into six groups. GPS $(50,100,200 \mathrm{mg} / \mathrm{kg})$, colchicine $(1 \mathrm{mg} / \mathrm{kg})$ or vehicle $(0.9 \%$ normal saline) were applied intraperitoneal. $1 \mathrm{~h}$ after pretreatment with colchicine and GPS, all groups were injected with MSU crystals (0.5 mg MSU crystals dissolved in $0.02 \mathrm{ml}$ PBS) into the instep of the left hind leg under isoflurane anesthesia, while the control group 

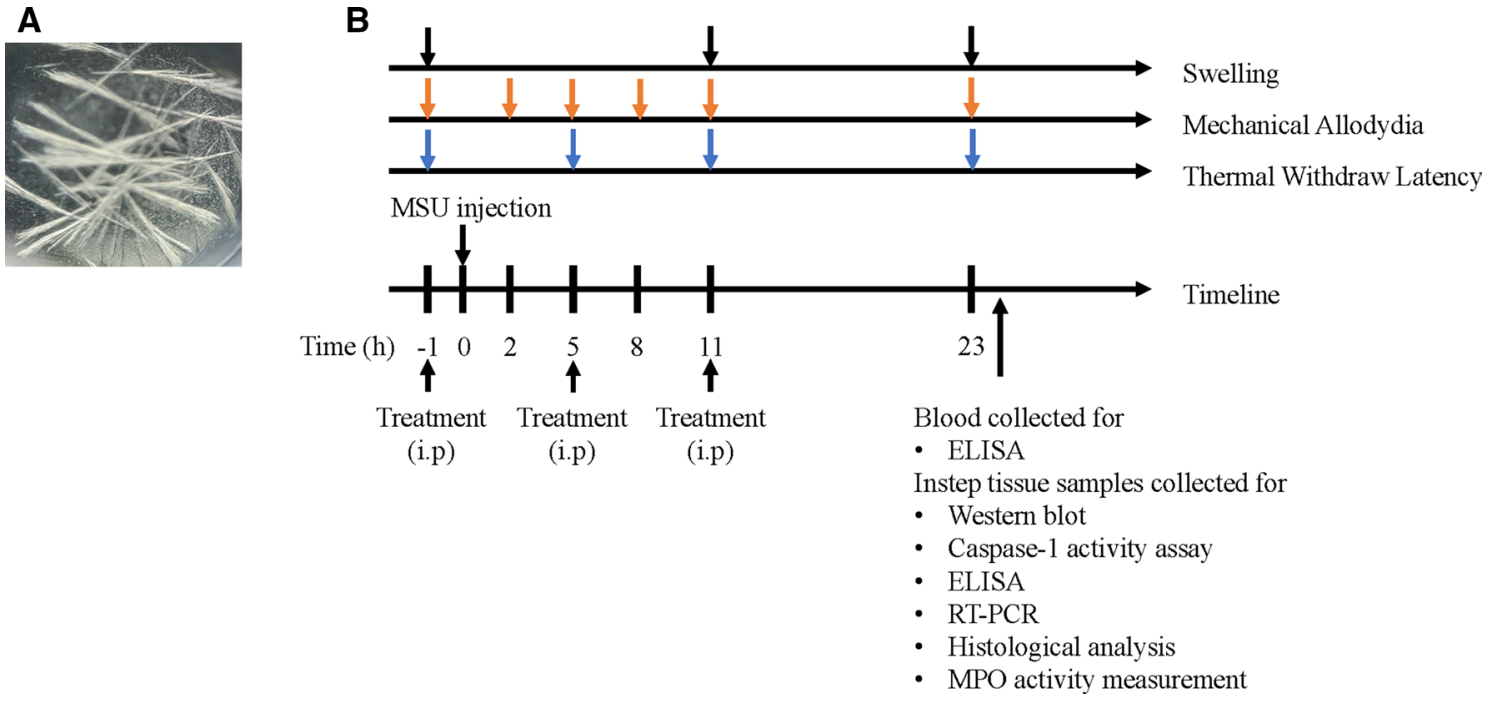

Fig. 1 The structure of MSU crystals and experimental protocol. a The structure of MSU crystals in polarizing microscope. b The experimental protocol: MSU crystals ( $0.5 \mathrm{mg}$ MSU in $20 \mu \mathrm{L}$ PBS) or PBS $(20 \mu \mathrm{L})$ was applied into the instep of right paw to establish the acute gouty arthritis model or control group. Swelling was measured at $1 \mathrm{~h}$ before MSU injection and 11, $23 \mathrm{~h}$ after model establishment.

was injected with the same volume of PBS. Treatment with GPS was conducted repeatedly at 5 and $11 \mathrm{~h}$ after MSU injection.

\section{Evaluation of swelling}

To assay the inflammation response of the instep, the circumference of the instep was measured by a graduated rope. Overall, a total of three measurements was conducted in the entire experiment, and the measurement result before MSU stimulation was used as the standard circumference. The next two measurements were performed at 11 and $23 \mathrm{~h}$ after the injection of MSU. Swelling index $=$ (circumference of the instep after MSU stimulation - standard circumference)/standard circumference.

\section{Evaluation of mechanical allodynia}

Mice were individually placed in the transparent glass boxes for 15-20 min to adapt to the environment before evaluation. In the bottom of the boxes, Von Frey calibrated filaments (UGO Basile, Italy) were used to detect the plantar surface of the left hind leg through the wire mesh, bending the filaments slightly for 3-5 s with enough force. Increasing filament stiffness if mice had no reaction or decreasing filament stiffness if mice appeared abrupt withdraw of the paw, licking or vigorously shaking. The intensity of the pressure was recorded. The up-down testing paradigm and nonparametric Dixon test were performed to calculate the
Mechanical allodynia was detected $1 \mathrm{~h}$ before MSU injection and 2, $5,8,11,23 \mathrm{~h}$ after MSU injection. The measurement of thermal withdraw latency was conducted at $1 \mathrm{~h}$ before MSU injection and 5, 11, $23 \mathrm{~h}$ after model establishment. Mice were killed to collect blood and instep tissue for Western blot, Caspase-1 activity assay, ELISA, RTPCR, Histological analysis, and MPO activity measurement

50\% paw withdrawal threshold (PWT) [22, 23]. The baseline of PWT was measured $1 \mathrm{~h}$ before the injection of MSU, and mechanical allodynia were frequently assessed at 2, 5, 8, 11 , and $23 \mathrm{~h}$ after the injection of MSU to explore the time effect of GPS and MSU.

\section{Evaluation of thermal withdraw latency}

Plantar test (UGO Basile, Varese, Italy) was applied to test thermal sensitivity as described previously [24]. Mice were placed in glass boxes individually for $15 \mathrm{~min}$ to acclimate the environment before the test. In brief, the hind paw was exposed to the radiant emission by the light source. The time of paw licking or withdrawal, which were considered as a response to avoid thermal pain, was recorded. The cut-off time was set as $30 \mathrm{~s}$ to avoid burns on the plantar skin of mice. We conducted three measurements with 10-min interval and the average of the three measurements was regarded as the final result. We performed tests at $1 \mathrm{~h}$ before and 5 , $11,23 \mathrm{~h}$ after the injection of MSU, respectively.

\section{Blood processing}

After mice were anesthetized by $1 \%$ sodium pentobarbital, blood samples were collected and kept at room temperature for about $1 \mathrm{~h}$ to ensure the separation of serum. Then the serum was collected through centrifugation at $5000 \mathrm{rpm}$ for $15 \mathrm{~min}$ at $37^{\circ} \mathrm{C}$. Serum samples were used for the cytokines assays for ELISA and stored at $-80^{\circ} \mathrm{C}$ before other assays. 


\section{Histological analysis}

The instep tissue samples were collected after last evaluation, and fixed in the 4\% paraformaldehyde solution. Then, the samples were decalcified for 20 days with EDTA and embedded with paraffin. Subsequently, the paraffin sections were stained with hematoxylin and eosin for conventional morphological assessment using a light microscope on $40 \times$ objective.

\section{Myeloperoxidase (MPO) activity measurement}

The MPO Detection Kit (Nanjing JianCheng Bio Ins, China) was used to evaluate the quantification of neutrophil infiltration in the paw tissue as described previously [25]. Briefly, mice were anaesthetized, and the paw tissue was homogenized and centrifuged at $12,000 \mathrm{r} / \mathrm{min}$ at $4{ }^{\circ} \mathrm{C}$ for $20 \mathrm{~min} .10 \mu \mathrm{L}$ of the supernatant was transferred into pbs

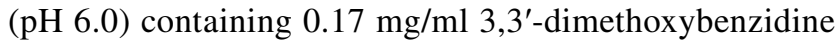
and $0.0005 \% \mathrm{H}_{2} \mathrm{O}_{2}$. MPO can catalyze the redox reaction of hydrogen peroxide with 3,3',5,5'-tetramethylbenzidine, which then produce a yellow compound. MPO activity was calculated by measuring the value of product in $460 \mathrm{~nm}$ with spectrophotometer and expressed as units per gram of total protein $(\mathrm{u} / \mathrm{g})$.

\section{Caspase- 1 activity assay}

The Caspase-1 Activity Kit (Beyotime Institute of Biotechnology, Nanjing, China) was used to assay the activity of Caspase-1 according to the manufacturer's protocol. This assay is based on the function of Caspase-1 that can convert acetyl-Tyr-Val-Ala-Asp p-nitroanilide (Ac-YVAD-pNA) into the yellow formazan product p-nitroanilide (p-NA). Tissue lysates were centrifuged at $14,000 \mathrm{~g}$ for $10 \mathrm{~min}$, and the protein concentrations were determined by the Bradford protein assay. A total of $50 \mu \mathrm{g}$ of protein was incubated in a 96-well microtiter plate with $20 \mathrm{ng}$ of Ac-DEVD-pNA overnight at $37{ }^{\circ} \mathrm{C}$. The absorbance value of pNA at $405 \mathrm{~nm}$ was measured using a 96-well plate reader. The increase of pNA value indicates the activation of caspase-1.

\section{Western blot}

Paw samples and cells were lysed in a RIPA buffer containing $1 \mathrm{mM}$ phenylmethylsulfonyl fluoride (PMSF) and further centrifugated at $14,000 \times g$ for $15 \mathrm{~min}$ at $4{ }^{\circ} \mathrm{C}$. The supernatant was obtained and its total protein concentration was measured by a BCA protein assay kit. Protein was loaded and separated by $8-12 \%$ sodium dodecyl sulfate-polyacrylamide gel electrophoresis (SDS-PAGE), then polyvinylidene fluoride (PVDF) membranes were applied to transfer protein. The membranes were blocked with $5 \%$ (w/v) non-fat milk in TBST buffer on the shaker for $2 \mathrm{~h}$ at room temperature followed by incubation with corresponding primary antibodies (1:1000 dilution) overnight at $4{ }^{\circ} \mathrm{C}$, and incubated with HRP-conjugated secondary antibodies (1:4000 dilution). Finally, antigen-antibody complexes were detected by ECL chemiluminescent substrate. The levels of protein expression are normalized to the density of $\beta$-actin.

\section{Real-time quantitative PCR}

The paw tissue samples at $23 \mathrm{~h}$ after the establishment of acute gouty arthritis and cells were collected and extracted in $1 \mathrm{~mL}$ Trizol reagent (Invitrogen, USA) for total RNA followed by centrifugation at $12,000 \mathrm{rpm}$ for $10 \mathrm{~min}$ at $4{ }^{\circ} \mathrm{C}$. The purity and concentration of the samples were measured using a spectrophotometer, and the standard of wavelength absorption ratio $(260 / 280 \mathrm{~nm})$ was set between 1.8 and 2.0. cDNA was obtained using HiScript III RT SuperMix (Vazyme Biotech Co., Ltd. China) according to the manufacturer's instructions. Gene expression was determined by Stratagene MX3000P using ChamQ Universal SYBR qPCR Master Mix (Vazyme Biotech Co., Ltd. China). Relative mRNA expression was calculated using $2^{-\Delta \Delta C t}$ method and GAPDH was used as reference gene. The qPCR primers are listed in Table 1.

\section{Enzyme-linked immunosorbent assay}

Serum and paw tissue were collected to detect the quantity of TNF- $\alpha$ (ZC-39024), IL-1 $\beta$ (ZC-37974), IL-6 (ZC37988), and IL-18 (ZC-37973) using corresponding ELISA kits according to the manufacturer's instructions (ZCIBIO Technology Co. Ltd).

\section{Statistical analyses}

Data were analyzed with GraphPad Prism 7 (GraphPad Software, La Jolla, CA, USA) and presented as the mean \pm SD. Statistical significance was determined by Student's $t$ test or one-way analysis of variance (ANOVA) followed by Bonferroni test. $P<0.05$ was considered statistically significant.

Table 1 Sequences of the primers used for qPCR

\begin{tabular}{ll}
\hline Genes & Sequences of primers \\
\hline NLRP3 & F: 5' GCCGTCTACGTCTTCTTCCTTTCC 3' \\
& R: 5' CATCCGCAGCCAGTGAACAGAG 3' \\
ASC & F: 5' ACAATGACTGTGCTTAGAGACA 3' \\
& R: 5' CACAGCTCCAGACTCTTCTTTA 3' \\
Caspase-1 & F: 5' AGAGGATTTCTTAACGGATGCA 3' \\
& R: 5' TCACAAGACCAGGCATATTCTT 3' \\
GAPDH & F: 5' CTCTCTGCTCCTGTTCGACAG 3' \\
& R: 5' GTGTAATCATATTGGAACATGTAG 3' \\
\hline
\end{tabular}




\section{Results}

\section{The therapeutic effects of GPS on MSU-induced pain and swelling}

Pain and swelling are the remarkable symptoms in patients with acute gouty arthritis in clinic [26]. Therefore, we established acute gouty arthritis model induced by MSU to analyze the effects of GPS on these signs. Evaluations were conducted at different times (Fig. 1b). We took paw swelling as an indicator of the effects of GPS. Figure 2a, b showed that GPS and colchicine relieve paw swelling induced by MSU. Compared with MSU + Veh group, $100 \mathrm{mg} / \mathrm{kg}$ and $200 \mathrm{mg} / \mathrm{kg}$ GPS groups showed significant inhibition on instep swelling index (Fig. 2c). Next, we evaluated the effects of GPS on MSU-induced pain. The results showed that the severity of pain induced by MSU achieved its peak at $8 \mathrm{~h}$ after stimulus. And $100 \mathrm{mg} / \mathrm{kg}$, $200 \mathrm{mg} / \mathrm{kg}$ GPS had analgesic effect on mechanical hyperalgesia, while the $50 \mathrm{mg} / \mathrm{kg}$ GPS did not show (Fig. 2d, e).
Furthermore, MSU-induced a severe thermal hyperplasia, which was inhibited by the treatment of GPS with $100 \mathrm{mg} /$ $\mathrm{kg}$ and $200 \mathrm{mg} / \mathrm{kg}$ (Fig. 2f, g).

\section{GPS inhibited NLRP3 inflammasome activation in vivo and vitro studies}

To further investigate whether the mechanism of GPS to treat acute gouty arthritis is related to NLRP3 inflammasome, we first performed western blot to analyze the activation of NLRP3 inflammasome in vivo study. After MSU stimulation, the expressions of NLRP3, ASC, and Caspase-1 were upregulated. But these effects were significantly inhibited by GPS (Fig. 3a-c). Next, we examined the effects of GPS on NLRP3 inflammasome activation by qPCR. Consistent with aforementioned results, the results of qPCR demonstrated that GPS significantly attenuated the overexpressions of NLRP3, ASC, and Caspase-1 mRNA in paw tissues induced by MSU (Fig. 3d-f). We further used the Caspase-1 Activity Kit to verify our results. Coherently, the

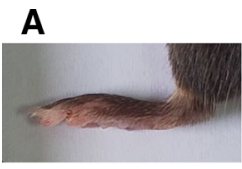

Control

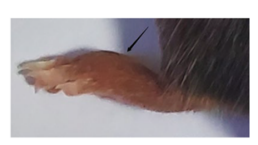

MSU+Veh

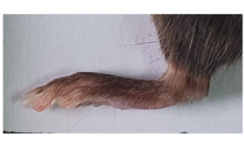

MSU+Colchicine

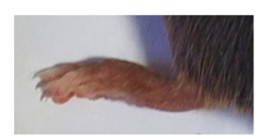

$\mathrm{MSU}+50 \mathrm{mg} / \mathrm{kg}$ GPS

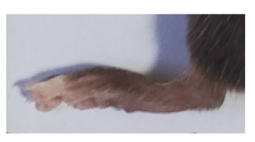

$\mathrm{MSU}+100 \mathrm{mg} / \mathrm{kg}$ GPS

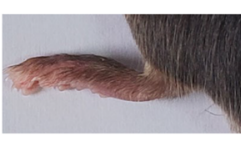

$\mathrm{MSU}+200 \mathrm{mg} / \mathrm{kg}$ GPS
B
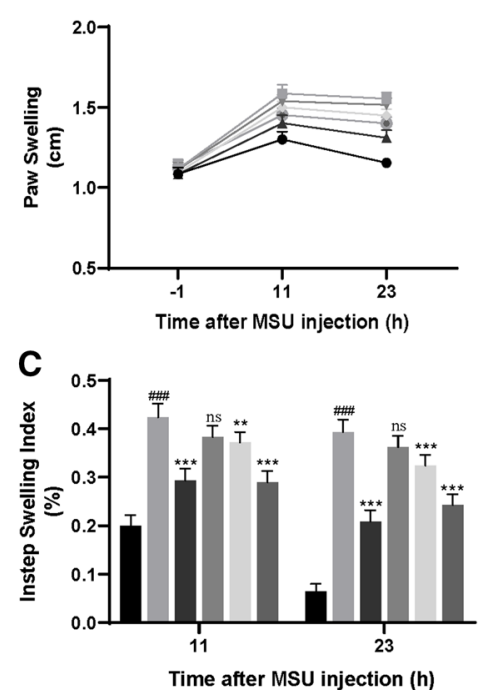

D

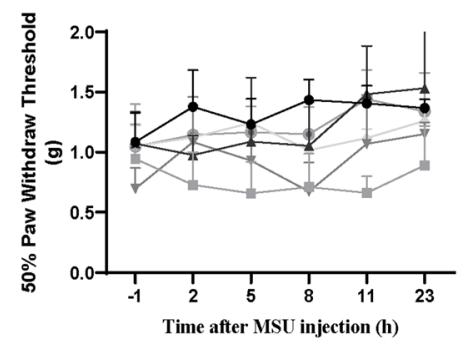

E

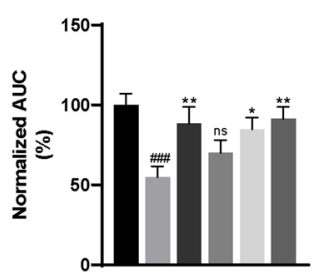

$\mathbf{F}$

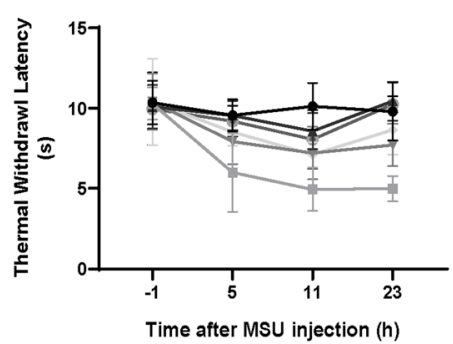

G

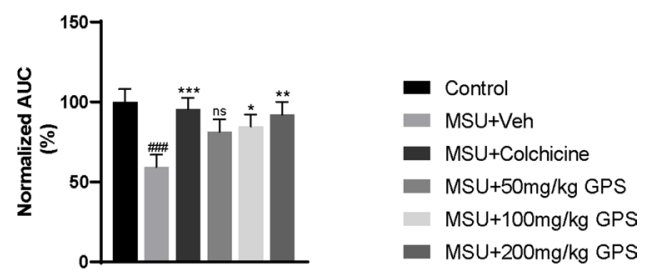

Fig. 2 Effects of GPS on swelling and pain in acute gouty arthritis induced by MSU. a Representative photographs of ankles $24 \mathrm{~h}$ after MSU injection. Black arrow indicates the injected place. b Time courses of the effects of colchicine and different doses of GPS on paw swelling. $n=6$ mice per group. c Comparison of the instep swelling indexes at different points after MSU injection in each group. d, $\mathbf{f}$ Time course of the effects of colchicine and different doses of
GPS on mechanical and thermal allodynia. e, g Normalized AUC of d, f. Values are expressed as means \pm SD. ${ }^{\# \#} p<0.001$ vs. the control group; $* p<0.05$ vs. the MSU + Veh group; $* * p<0.01$ vs. the MSU + Veh group. ${ }^{* *} p<0.001$ vs. the MSU + Veh group. ns, not significantly different from MSU + Veh group; one-way or two-way ANOVA followed by Tukey's post hoc test 

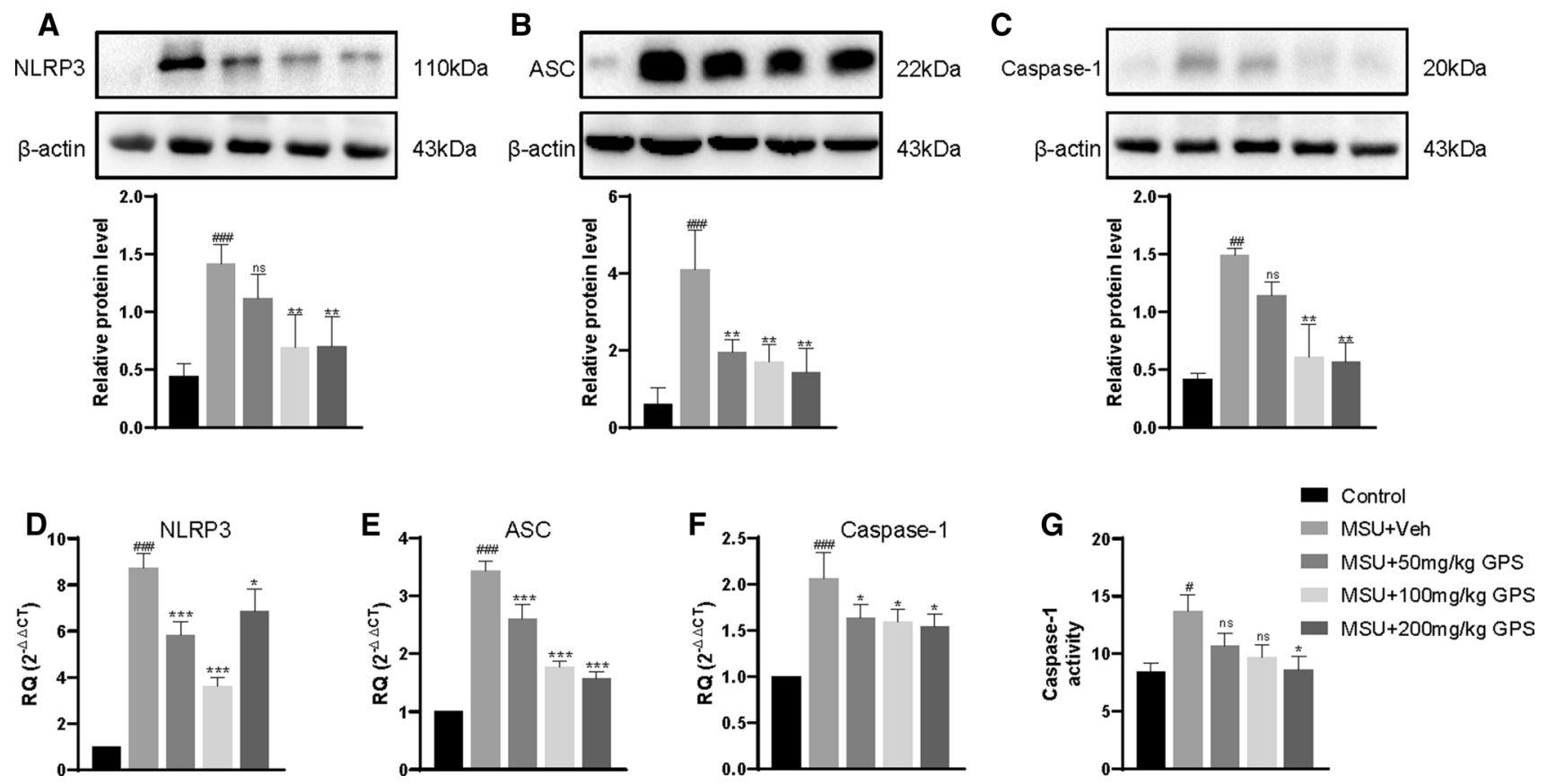

Fig. 3 GPS inhibited the activation of NLRP3 inflammasome in paw tissue of acute gouty arthritis. NLRP3 (a), ASC (b) and Caspase-1 (c) protein expressions were evaluated $24 \mathrm{~h}$ after MSU injection. $\mathrm{n}=6$ mice per group. Measurements of NLRP3/ $\beta$-actin, ASC $/ \beta$-actin, and Caspase- $1 / \beta$-actin were quantified by Image $J$ software. $\mathbf{d}-\mathbf{f}$ The mRNA levels of NLRP3, ASC, and Caspase-1 in paw tissue were determined by qPCR. $\mathbf{g}$ The activity of Caspase- 1 in paw tissues of

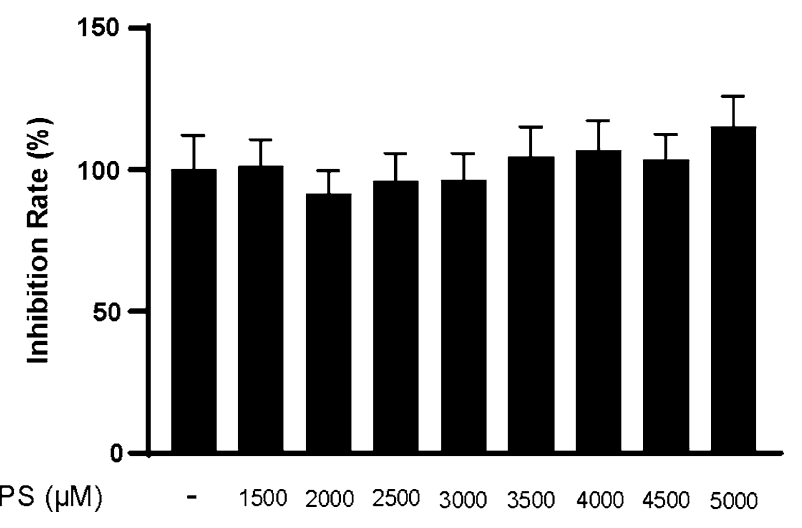

Fig. 4 Effects of GPS-induced cytotoxicity in RAW264.7 macrophages. All data were presented as mean \pm SD. There was no difference between groups

results showed that GPS can downregulate the activity of caspase-1 (Fig. 3g).

Furthermore, we conducted in vitro study to confirm the inhibition of GPS on the activation of NLRP3 inflammasome. To choose the optimal concentrations of GPS in this study, the CCK-8 assay was performed. The results demonstrated that GPS showed no significant toxicity to RAW264.7 macrophages at concentration below $5000 \mu \mathrm{M}$
MSU-induced acute gouty arthritis mice was determined by the Caspase-1 Activity Kit. Values are expressed as means \pm SD. ${ }^{\#} p<0.05$ vs. the control group; ${ }^{\# \#} p<0.01$ vs. the control group; ${ }^{\# \#} p<0.001$ vs. the control group; * $p<0.05$ vs. the MSU + Veh group; $* * p<0.01$ vs. the MSU + Veh group. ${ }^{* *} p<0.001$ vs. the MSU + Veh group. ns, not significantly different from MSU + Veh group

after $24 \mathrm{~h}$ (Fig. 4). Similarly, we assessed the level of crucial components of the NLRP3 inflammasome in RAW264.7 macrophages. The results showed that the expressions of NLRP3, ASC, and Caspase-1 were inhibited by GPS in dose-dependent (Fig. 5a-c). The mRNA levels of NLRP3, ASC, and Caspase-1 induced by LPS and MSU were also inhibited by GPS (Fig. 5d-f).

\section{The inhibition of GPS on the release of pro-inflammatory cytokines and neutrophil infiltration}

Considering the important effect of pro-inflammatory cytokines on the development of acute gouty arthritis, we explored whether GPS could inhibit the release of IL- $1 \beta$, IL-6, IL-18, and TNF- $\alpha$. We analyzed the expression levels of IL- $1 \beta$, TNF- $\alpha$, IL- 6 in serum, and found that GPS decreased the expressions of TNF- $\alpha$ (Fig. 6a), IL-6 (Fig. 6b), and IL-1 $\beta$ (Fig. $6 c$ ). Then, the pro-inflammatory cytokines in the paw tissue were analyzed. The expression levels of IL-1 $\beta$, IL-6, and IL-18 were inhibited by GPS except the group of $50 \mathrm{mg} / \mathrm{kg}$ GPS (Fig. $6 \mathrm{~d}-\mathrm{f}$ ), and GPS had no effect on the expression level of TNF- $\alpha$ (Fig. $6 \mathrm{~g}$ ).

We further examined the effects of GPS on inflammatory cell infiltration induced by MSU in paw tissue. MSU 

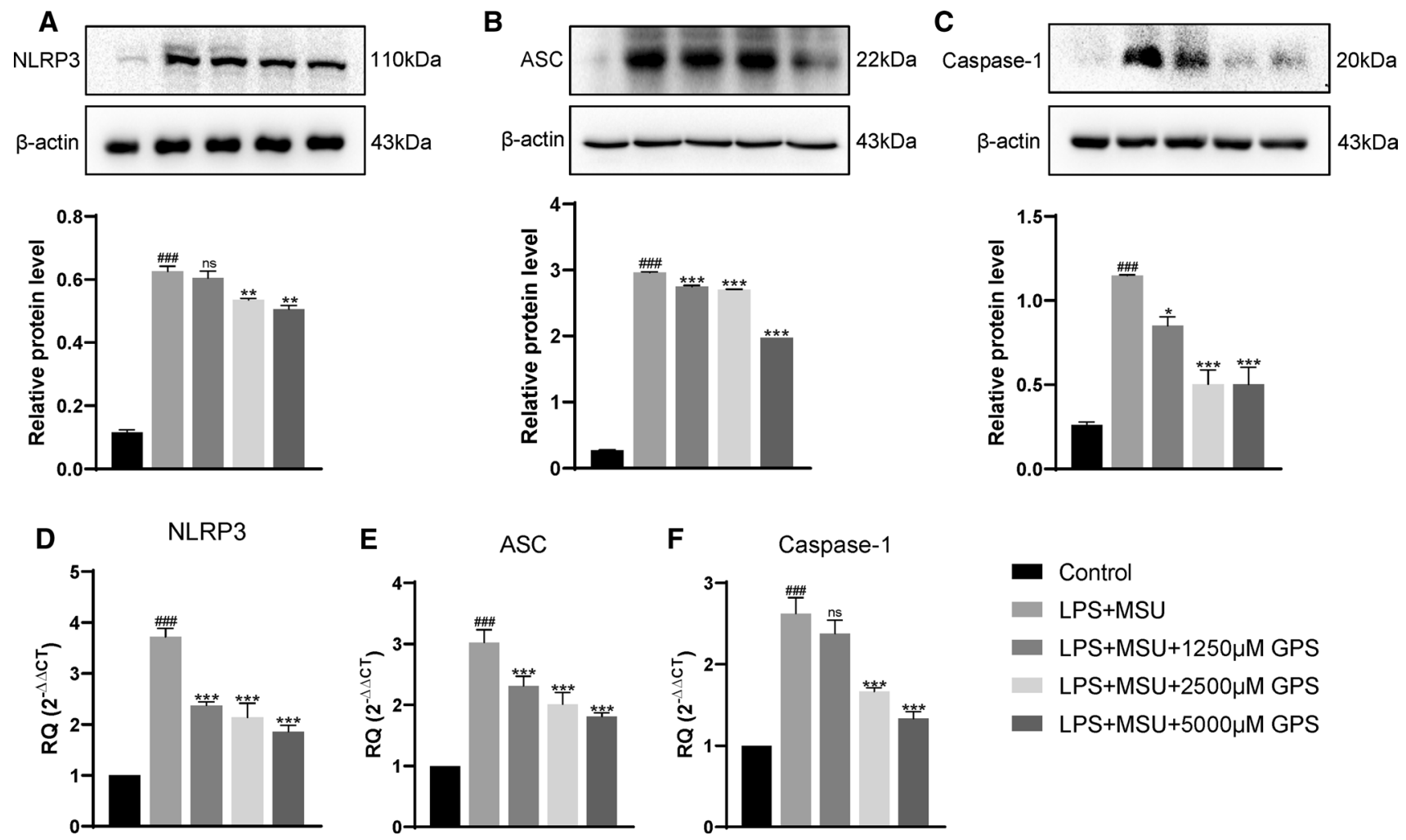

Fig. 5 GPS inhibited the activation of NLRP3 inflammasome in RAW264.7 macrophages induced by LPS-MSU. NLRP3 (a), ASC (b) and Caspase-1 (c) protein expressions were evaluated. Measurements of NLRP3/ $\beta$-actin, ASC $/ \beta$-actin and Caspase- $1 / \beta$-actin were quantified by Image $\mathbf{J}$ software. (D,E,F) The mRNA levels of NLRP3,
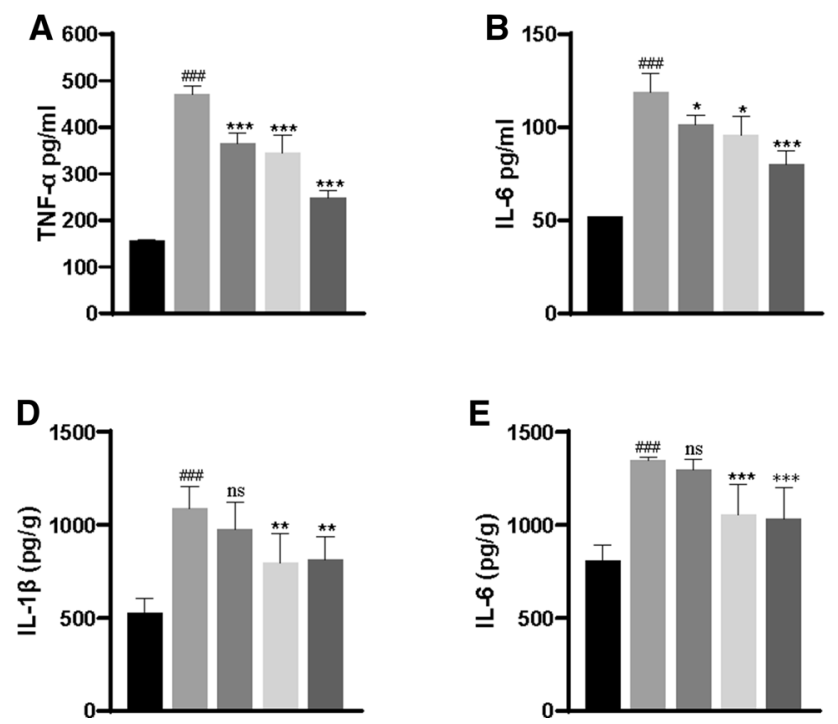

Fig. 6 The inhibition of GPS on the release of pro-inflammatory cytokines, including IL-1 $\beta$, IL- 6 , IL-18, and TNF- $\alpha$ in serum and paw tissue. a, b The TNF- $\alpha$ and IL- 6 levels were markedly decreased by the treatment of GPS than the control group in serum. c-e The IL-1 $\beta$, IL-6, and IL-18 levels were significantly reduced in
ASC, Caspase-1 in RAW264.7 macrophages were determined by qPCR. Values are expressed as means \pm SD. ${ }^{\# \# \# p} p<0.001$ vs. the control group; $* p<0.05$ vs. the MSU + Veh group; $* * p<0.01$ vs. the MSU + Veh group. $* * * p<0.001$ vs. the MSU + Veh group. ns, not significantly different from MSU + Veh group
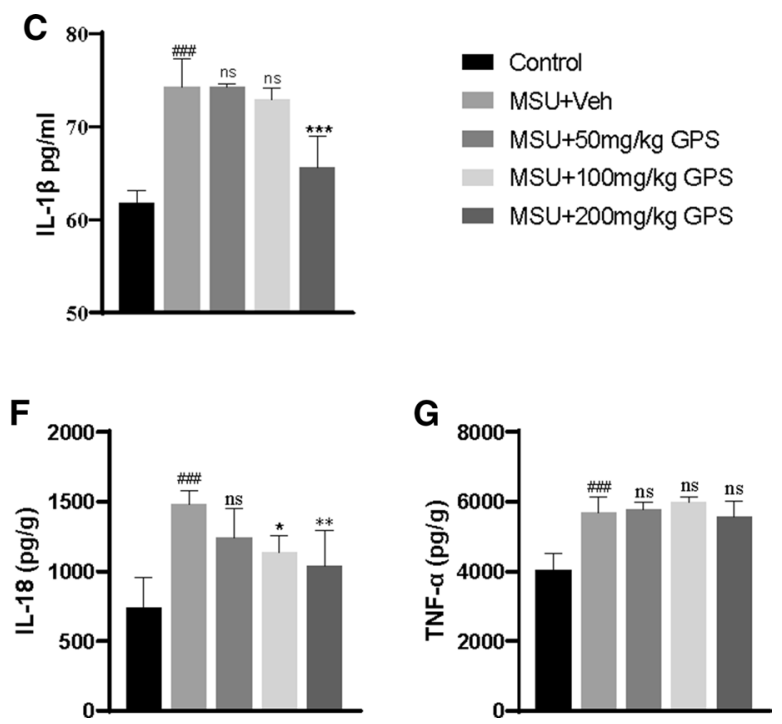

$\mathrm{MSU}+100 \mathrm{mg} / \mathrm{kg}$ and $\mathrm{MSU}+200 \mathrm{mg} / \mathrm{kg}$ groups in paw tissue. Values are expressed as means \pm SD. ${ }^{\# \# p} p<0.001$ vs. the control group; $* p<0.05$ vs. the MSU + Veh group; $* * p<0.01$ vs. the MSU + Veh group. ${ }^{* * *} p<0.001$ vs. the MSU + Veh group. ns, not significantly different from MSU + Veh group 
significantly induced the inflammatory cells infiltration in paw tissue. Furthermore, animals treated with GPS and colchicine progressed to less paw tissue lesion, as evidenced by significantly decreased infiltration of inflammatory cells in the instep of paw (Fig. 7a). Previous study [27] demonstrated that neutrophil depletion alleviates inflammation, indicating the significance of inhibition on the recruitment of neutrophil. Therefore, Myeloperoxidase (MPO) assay was performed to evaluate the effects of GPS on neutrophil infiltration in paw tissue, GPS and colchicine both significantly attenuated MSU-induced neutrophil infiltration in paw tissue (Fig. 7b).

\section{Discussion}

In the present study, we parsed out the role of GPS in the model of acute gouty arthritis induced by MSU and in the RAW264.7 macrophages induced by LPS-MSU. The model aforementioned shares canonical characteristics of gout flares in humans. The injection of MSU-induced swelling, severe pain, pro-inflammatory cytokines release, the infiltration of neutrophil and the activation of NLRP3 inflammasome. Whereas, these effects were alleviated by the treatment with GPS. The activation of NLRP3 inflammasome in RAW264.7 cells induced by LPS-MSU was also inhibited by GPS. Above results demonstrated that GPS is a promising treatment for acute gouty arthritis.

GPS has been reported to have an obvious inhibition on swelling in arthritis induced by adjuvant [28]. Research [29] also demonstrated that GPS has an analgesic effect on
A

Control

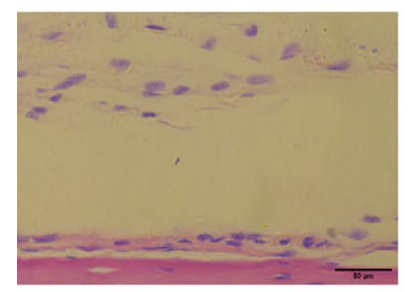

$\mathrm{MSU}+50 \mathrm{mg} / \mathrm{kg}$ GPS

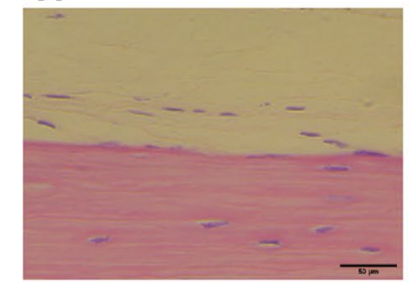

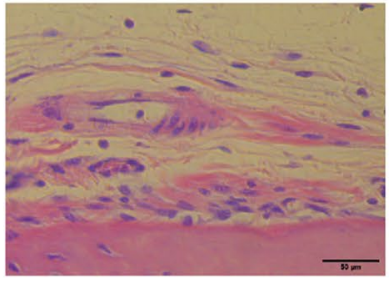

MSU+Veh

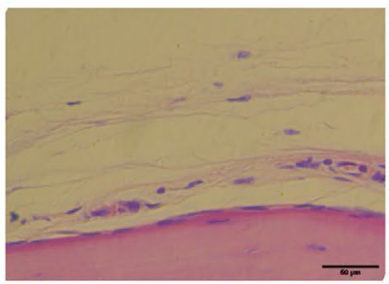

$\mathrm{MSU}+100 \mathrm{mg} / \mathrm{kg}$ GPS
Fig. 7 Effects of GPS on inflammatory cell infiltration in instep tissues in the model of acute gouty arthritis induced by MSU. a H\&E staining $(\times 400)$ of inflammatory cells in instep tissues. b Myeloper- mechanical, thermal, cold hyperalgesia in CCI model. And the effects of GPS on mechanical, thermal pain induced by MSU were indicated by our findings. Furthermore, we compared the effects of GPS with colchicine and found that they share similar effectiveness. Here, we demonstrated that GPS could effectively attenuate swelling and pain in acute gouty arthritis induced by MSU, which provides the first evidence displaying the therapeutic effects of GPS on acute gouty arthritis.

MSU crystals induce an inflammatory response, which is dependent on the activation of NLRP3 inflammasome [30]. Research [31] reported that $\mathrm{NLRP3}^{-/-}, \mathrm{ASC}^{-/-}$, and Caspase- $1^{-/}$mice alleviate MSU-induced ankle swelling as compared to WT mice. GPS has been shown to possess the inhibition on the expression of NLRP3, ASC, and Caspase- 1 in adjuvant-induced arthritis [32]. In the present study, MSU-induced an obvious increase in NLRP3, ASC, Caspase-1, and IL-1 $\beta$, and these growths were curbed by GPS treatment. Together, these results demonstrated that GPS is able to attenuate gouty arthritis via inhibiting NLRP3 inflammasome activation. However, further explorations of the mechanism of GPS on NLRP3 inflammasome are required.

Pro-inflammatory cytokines have been implicated in initiation and amplification of the gout flare, such as IL-1 $\beta$, IL-6, and TNF- $\alpha$ [12]. The activation of NLRP3 inflammasome induces the release of IL- $1 \beta$ and IL-18. Afterwards, IL-1 $\beta$ promotes the expression of pro-inflammatory cytokines, including TNF- $\alpha$ and IL-6, leading to neutrophil infiltration [33]. In turn, neutrophils interact with MSU crystals and induce the release of IL-1, IL-6, TNF- $\alpha$, and other cytokines,
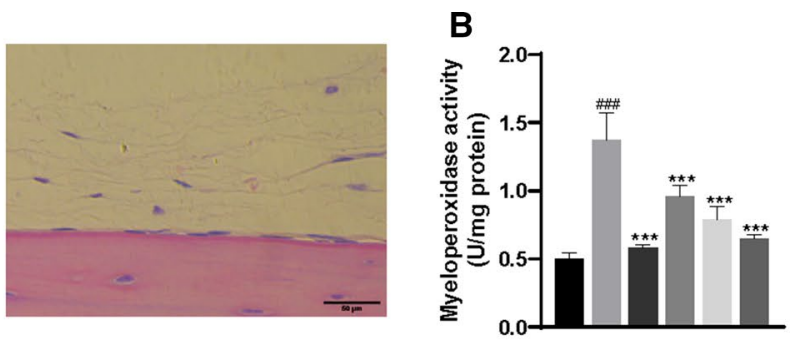

MSU+Colchicine
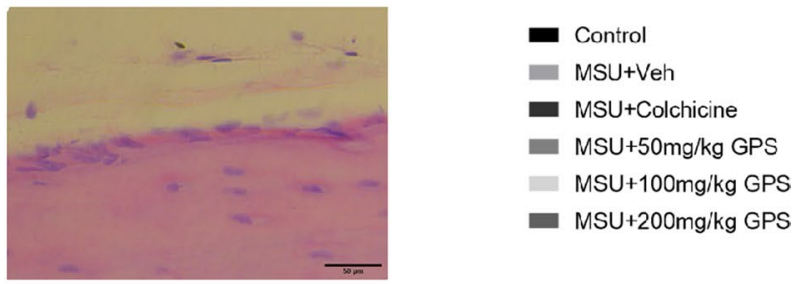

$\mathrm{MSU}+200 \mathrm{mg} / \mathrm{kg}$ GPS

oxidase (MPO) activity was determined in instep tissues. $n=6$ mice per group. Values are expressed as means \pm SD. ${ }^{\# \#} p<0.001$ vs. the control group; ${ }^{* * *} p<0.001$ vs. the MSU + Veh group 
resulting in pain and erythema related to gout flare [34, 35]. Inhibition on TNF- $\alpha$ and IL- $1 \beta$ has been demonstrated to reduce neutrophil infiltration and pain in rheumatic disease $[30,36]$. IL-18 is deemed to elevate cell adhesion molecules for leukocyte trafficking and induces chemokine production [37]. Therefore, it is effective to inhibit neutrophil infiltration induced by MSU and the release of pro-inflammatory cytokines in the management of acute gouty arthritis. Consistent with previous results [38], we verified that GPS can decrease the levels of TNF- $\alpha$, IL- 6 in serum and inhibit the release of IL-1 $\beta$, IL- 6 , and IL-18 in the paw tissue. The neutrophil infiltration induced by MSU was also alleviated by GPS.

In conclusion, the present study indicated that GPS can attenuate MSU-induced pain, swelling, and neutrophil infiltration via inhibiting the release of pro-inflammatory cytokines. We further demonstrated that the effects of GPS are related to the inactivation of NLRP3 inflammasome in vivo and vitro study. To our knowledge, this is the first time to explore the mechanism of GPS in treating acute gouty arthritis. Therefore, GPS may be a novel effective strategy in treating acute gouty arthritis based on analgesic and anti-inflammatory properties.

Acknowledgements This study was supported by the National Natural Science Foundation of China no. 81673741 and Postgraduate Research and Practice Innovation Program of Jiangsu Province no. KYCX21_1629.

Open Access This article is licensed under a Creative Commons Attribution 4.0 International License, which permits use, sharing, adaptation, distribution and reproduction in any medium or format, as long as you give appropriate credit to the original author(s) and the source, provide a link to the Creative Commons licence, and indicate if changes were made. The images or other third party material in this article are included in the article's Creative Commons licence, unless indicated otherwise in a credit line to the material. If material is not included in the article's Creative Commons licence and your intended use is not permitted by statutory regulation or exceeds the permitted use, you will need to obtain permission directly from the copyright holder. To view a copy of this licence, visit http://creativecommons.org/licenses/by/4.0/.

\section{References}

1. Khanna D, Fitzgerald JD, Khanna PP et al (2012) 2012 American College of Rheumatology guidelines for management of gout. Part 1: systematic nonpharmacologic and pharmacologic therapeutic approaches to hyperuricemia. Arthritis Care Res (Hoboken) 64:1431-1446. https://doi.org/10.1002/acr.21772

2. Dehlin M, Jacobsson L, Roddy E (2020) Global epidemiology of gout: prevalence, incidence, treatment patterns and risk factors. Nat Rev Rheumatol 16:380-390. https://doi.org/10.1038/ s41584-020-0441-1

3. Zhang Y, Su H, Zhang J, Kong J (2019) The effects of ginsenosides and anserine on the Up-regulation of renal aquaporins 1-4 in hyperuricemic mice. Am J Chin Med 47:1133-1147. https:// doi.org/10.1142/S0192415X19500587

4. Chhana A, Lee G, Dalbeth N (2015) Factors influencing the crystallization of monosodium urate: a systematic literature review. BMC Musculoskelet Disord 16:296. https://doi.org/10.1186/ s12891-015-0762-4

5. Dalbeth N, Choi HK, Joosten LAB et al (2019) Gout. Nat Rev Dis Primers 5:69. https://doi.org/10.1038/s41572-019-0115-y

6. Schumacher HR (1975) Pathology of the synovial membrane in gout. Light and electron microscopic studies. Interpretation of crystals in electron micrographs. Arthritis Rheum 18:771-782. https://doi.org/10.1002/art.1780180722

7. Terkeltaub R (2010) Update on gout: new therapeutic strategies and options. Nat Rev Rheumatol 6:30-38. https://doi.org/10.1038/ nrrheum.2009.236

8. So AK, Martinon F (2017) Inflammation in gout: mechanisms and therapeutic targets. Nat Rev Rheumatol 13:639-647. https://doi. org/10.1038/nrrheum.2017.155

9. Latz E, Xiao TS, Stutz A (2013) Activation and regulation of the inflammasomes. Nat Rev Immunol 13:397-411. https://doi.org/ $10.1038 /$ nri3452

10. Kelley N, Jeltema D, Duan Y, He Y (2019) The NLRP3 inflammasome: an overview of mechanisms of activation and regulation. Int J Mol Sci. https://doi.org/10.3390/ijms20133328

11. Dinarello CA (2009) Immunological and inflammatory functions of the interleukin-1 family. Annu Rev Immunol 27:519-550. https://doi.org/10.1146/annurev.immunol.021908.132612

12. Dinarello CA (2011) Interleukin-1 in the pathogenesis and treatment of inflammatory diseases. Blood 117:3720-3732. https://doi. org/10.1182/blood-2010-07-273417

13. Wu ZC, Xue Q, Zhao ZL et al (2019) Suppressive effect of Huzhentongfeng on experimental gouty arthritis: an in vivo and in vitro study. Evid Based Complement Alternat Med 2019:2969364. https://doi.org/10.1155/2019/2969364

14. Chen F, Xie L, Kang R et al (2018) 11.Gentiopicroside inhibits RANKL-induced osteoclastogenesis by regulating NF-kappaB and JNK signaling pathways. Biomed Pharmacother 100:142146. https://doi.org/10.1016/j.biopha.2018.02.014

15. Hu ZQ, Wang H, Fu Y et al (2021) Gentiopicroside inhibits cell growth and migration on cervical cancer via the reciprocal MAPK/Akt signaling pathways. Nutr Cancer 73(8):1459-1470. https://doi.org/10.1080/01635581.2020.1801777

16. Yang HX, Shang Y, Jin Q et al (2020) Gentiopicroside ameliorates the progression from hepatic steatosis to fibrosis induced by chronic alcohol intake. Biomol Ther (Seoul) 28:320-327. https:// doi.org/10.4062/biomolther.2020.008

17. Zhao L, Ye J, Wu GT et al (2015) 14.Gentiopicroside prevents interleukin-1 beta induced inflammation response in rat articular chondrocyte. J Ethnopharmacol 172:100-107. https://doi.org/10. 1016/j.jep.2015.06.031

18. Niu YT, Zhao YP, Jiao YF et al (2016) Protective effect of gentiopicroside against dextran sodium sulfate induced colitis in mice. Int Immunopharmacol 39:16-22. https://doi.org/10.1016/j.intimp. 2016.07.003

19. Zhang N, Jiang Y, Mu F et al (2019) Gentiopicrin exerts antirheumatic effect in human fibroblast-like synoviocytes via inhibition of p38MAPK/NF-кB pathway. Cell Mol Biol. https://doi.org/ 10.14715/cmb/2019.65.6.14

20. Yang Y, Wang Z, Zhang L et al (2018) Protective effect of gentiopicroside from Gentiana macrophylla Pall. in ethanol-induced gastric mucosal injury in mice. Phytother Res PTR 32:259-266. https://doi.org/10.1002/ptr.5965

21. Fattori V, Zarpelon AC, Staurengo-Ferrari L et al (2018) Budlein A, a sesquiterpene lactone From Viguiera robusta, alleviates pain and inflammation in a model of acute gout arthritis in mice. Front Pharmacol 9:1076. https://doi.org/10.3389/fphar.2018.01076 
22. Dixon WJ (1980) Efficient analysis of experimental observations. Annu Rev Pharmacol Toxicol 20:441-462. https://doi.org/10. 1146/annurev.pa.20.040180.002301

23. Chaplan SR, Bach FW, Pogrel JW et al (1994) Quantitative assessment of tactile allodynia in the rat paw. J Neurosci Methods 53:55-63. https://doi.org/10.1016/0165-0270(94)90144-9

24. Austin PJ, Wu A, Moalem-Taylor G (2012) Chronic constriction of the sciatic nerve and pain hypersensitivity testing in rats. J Vis Exp JoVE. https://doi.org/10.3791/3393

25. Liu SH, Ma K, Xu XR, Xu B (2010) A single dose of carbon monoxide intraperitoneal administration protects rat intestine from injury induced by lipopolysaccharide. Cell Stress Chaperones 15:717-727. https://doi.org/10.1007/s12192-010-0183-0

26. Taylor WJ, Fransen J, Jansen TL et al (2015) Study for updated gout classification criteria: identification of features to classify gout. Arthritis Care Res 67:1304-1315. https://doi.org/10.1002/ acr.22585

27. Fattori V, Staurengo-Ferrari L, Zaninelli TH et al (2020) IL-33 enhances macrophage release of IL-1beta and promotes pain and inflammation in gouty arthritis. Inflamm Res 69:1271-1282. https://doi.org/10.1007/s00011-020-01399-x

28. Xie X, Li H, Wang Y et al (2019) Therapeutic effects of gentiopicroside on adjuvant-induced arthritis by inhibiting inflammation and oxidative stress in rats. Int Immunopharmacol 76:105840. https://doi.org/10.1016/j.intimp.2019.105840

29. Liu N, Li YX, Gong SS et al (2016) Antinociceptive effects of gentiopicroside on neuropathic pain induced by chronic constriction injury in mice: a behavioral and electrophysiological study. Can J Physiol Pharmacol 94:769-778. https://doi.org/10.1139/ cjpp-2015-0462

30. Amaral FA, Costa VV, Tavares LD et al (2012) NLRP3 inflammasome-mediated neutrophil recruitment and hypernociception depend on leukotriene B(4) in a murine model of gout. Arthritis Rheum 64:474-484. https://doi.org/10.1002/art.33355

31. Reber LL, Marichal T, Sokolove J et al (2014) Contribution of mast cell-derived interleukin-1beta to uric acid crystal-induced acute arthritis in mice. Arthritis Rheumatol 66:2881-2891. https:// doi.org/10.1002/art.38747

32. Wang M, Li H, Wang Y et al (2020) Anti-rheumatic properties of gentiopicroside are associated with suppression of ROS-NF-kappaB-NLRP3 axis in fibroblast-like synoviocytes and NF-kappaB pathway in adjuvant-induced arthritis. Front Pharmacol 11:515. https://doi.org/10.3389/fphar.2020.00515

33. Pope RM, Tschopp J (2007) The role of interleukin-1 and the inflammasome in gout: implications for therapy. Arthritis Rheum 56:3183-3188. https://doi.org/10.1002/art.22938

34. Liu X, Chen R, Shang Y et al (2008) Lithospermic acid as a novel xanthine oxidase inhibitor has anti-inflammatory and hypouricemic effects in rats. Chem Biol Interact 176:137-142. https://doi. org/10.1016/j.cbi.2008.07.003

35. Sabina EP, Nagar S, Rasool M (2011) A role of piperine on monosodium urate crystal-induced inflammation-an experimental model of gouty arthritis. Inflammation 34:184-192. https://doi. org/10.1007/s10753-010-9222-3

36. Amaral FA, Bastos LF, Oliveira TH et al (2016) Transmembrane TNF-alpha is sufficient for articular inflammation and hypernociception in a mouse model of gout. Eur J Immunol 46:204-211. https://doi.org/10.1002/eji.201545798

37. Chan AH, Schroder K (2020) Inflammasome signaling and regulation of interleukin-1 family cytokines. J Exp Med. https://doi.org/ 10.1084/jem.20190314

38. Wang Q, Zhou X, Yang L et al (2019) Gentiopicroside (GENT) protects against sepsis induced by lipopolysaccharide (LPS) through the NF-kappaB signaling pathway. Ann Transl Med 7:731. https://doi.org/10.21037/atm.2019.11.126

Publisher's Note Springer Nature remains neutral with regard to jurisdictional claims in published maps and institutional affiliations. 